\title{
MLSE Diversity Receiver for Partial Response CPM
}

\author{
Li Zhou* ${ }^{* \dagger}$, Philippa A. Martin*, Desmond P. Taylor*, Clive Horn ${ }^{\dagger}$ \\ * Department of Electrical and Computer Engineering \\ University of Canterbury, Christchurch, New Zealand \\ Email: \{p.martin,taylor\}@elec.canterbury.ac.nz \\ ${ }^{\dagger}$ Wireless Research Centre, University of Canterbury, Christchurch, New Zealand \\ Email: clive.horn@nzi3.com
}

\begin{abstract}
In this paper we consider the application of persurvivor processing and diversity techniques to a partial response continuous phase modulation (PR-CPM) maximum likelihood sequence estimation (MLSE) receiver design. In particular, we consider practical implementation in a public safety narrowband radio environment. Frequency pulse truncation and tilted phase are used to reduce the number of states in the PR-CPM trellis with little loss in real world performance. Selection, equal gain and maximum ratio combining techniques are also considered to assess the practical benefit in system deployment.
\end{abstract}

\section{INTRODUCTION}

Continuous phase modulation (CPM) maintains a constant envelope and phase continuity throughout transmission. These properties are ideal for wireless communications due to the good spectral efficiency coupled with excellent power efficiency [11]. Here, we consider partial response continuous phase modulation (PR-CPM) using a phase shaping filter with long correlation length. This lowers spectral side lobes, which reduces adjacent channel interference (ACI) and improves bandwidth efficiency [3]. This makes it attractive for narrow bandwidth operations, which typify the technology used for long range voice communications in the public safety environment. Unfortunately, PR-CPM has several drawbacks. Firstly, the complex nature of CPM with long correlation length means that conventional maximum likelihood sequence estimation (MLSE) receivers are costly to implement [5]. Practically this cost presents itself as memory, processing complexity and therefore power consumption. Any implementation of the receiver is sensitive to all these parameters. Secondly, the fading associated with moving vehicles compromises the reliability of conventional channel estimation techniques.

In this paper, we describe a design approach which allows a simplified implementation of a PR-CPM MLSE receiver. In particular, receiver complexity is significantly reduced by utilizing trellis state reduction, via the tilted phase approach [1], [8] and frequency pulse truncation [13]. Public safety operations commonly occur at speed and operation in the $800 \mathrm{MHz}$ band leads to fade rates requiring more careful attention to this element of the receiver design. To improve receiver reliability in a fading environment, a per-survivor processing (PSP) based approach [10], [4] to channel estimation is used. In addition, multiple receiver antennas and diversity combining are used to further combat the degradation due to the fast fading channel. The novelty lies in the integrated combination of PSP and diversity techniques in a practical, reduced-state PR-CPM MLSE based receiver design.

The paper is organized as follows. The transmitter, channels and PR-CPM are described in Section II. The receiver design is outlined in Section III. Simulation results are presented in Section IV and conclusions are drawn in Section V.

\section{System MOdEL}

We consider an uncoded PR-CPM system. The single- $h$ CPM signal at time $t$ is defined as [9], [12]

$$
s(t)=\sqrt{\frac{2 E}{T}} \cos \left[2 \pi f_{c} t+\theta(t ; \boldsymbol{I})+\theta_{n}\right], t \geq 0,
$$

where the phase state is defined as

$$
\theta_{n}=h \pi \sum_{k=-\infty}^{n-L} I_{k}
$$

and the correlative phase component is defined as

$$
\theta(t ; \boldsymbol{I})=2 \pi h \sum_{k=n-L+1}^{n-1} I_{k} q(t-k T), \quad n T \leq t \leq(n+1) T
$$

which is specified by the correlative state $\left(I_{n-1}, \cdots, I_{n-L+1}\right)$ consisting of component $M$-ary data symbols ${ }^{1}$ from the set $\pm 1, \pm 3, \cdots, \pm(M-1) . T$ is the symbol time, $E$ is symbol energy and $f_{c}$ is the carrier frequency. The phase response affecting the phase transition over $L$ symbol periods is given by

$$
q(t)=\int_{-\infty}^{t} g(\tau) d \tau= \begin{cases}0, & t<0 \\ \frac{1}{2}, & t>L T\end{cases}
$$

where $g(t)$ is the frequency pulse, which is a smooth pulse shape on the interval $(0, L T)$, normalized such that $\int_{-\infty}^{\infty} g(t) d t=1 / 2 . L$ is the duration of $g(t)$ in symbol periods and $h=m / p$ is the modulation index, where $m$ and $p$ are relatively prime positive integers.

We consider a CPM system with $L=4$ (ensuring a long correlation length to reduce ACI), $h=1 / 3$ (to conserve bandwidth at the cost of energy), $M=4$ (to ensure a good

\footnotetext{
${ }^{1} M$ is usually a power of 2 .
} 
joint energy-bandwidth tradeoff) and a frequency pulse defined as [15]

$$
g(t)=\left\{\begin{array}{cl}
\frac{1}{G}(\operatorname{sinc}(\lambda(t-L T / 2) / T)) & \\
\cdot\left(\cos ^{2}(\pi(t-L T / 2) / T / L)\right) & \text { for } t \in[0, L T] \\
0 & \text { elsewhere. }
\end{array}\right.
$$

where $G=2.60731391$ is a normalization factor and $\lambda=0.75$ is a modulation parameter, such that $q(t)=1 / 2$ for $t \geq L T$.

Here, we consider both additive white Gaussian noise $(A W G N)$ and Rayleigh fading channels. The received signal at time $n T$ for an AWGN channel is given by

$$
r(n T)=s(n T)+e(n T)
$$

and for the Rayleigh fading channel by

$$
r(n T)=h(n T) s(n T)+e(n T),
$$

where $h(n T)$ is the channel gain and $e(n T)$ is AWGN.

\section{PSP-BASED MLSE RECEIVER DESIGN}

We now consider the PSP-based MLSE receiver. The standard demodulator used for CPM is MLSE using the Viterbi algorithm (VA) [1]. We consider PR-CPM with a response length of $L=4$ symbol periods. Then, each CPM encoded symbol can be represented by a state $S=\left(\theta_{n}, I_{n-1}, I_{n-2}, I_{n-3}\right)$, where $I_{k} \in\{ \pm 1, \pm 3\}$ and $\theta_{n} \in\left\{0, \frac{\pi}{3}, \frac{2 \pi}{3}, \pi, \frac{4 \pi}{3}, \frac{5 \pi}{3}\right\}$. Since there are 64 combinations of $I_{n-1}, I_{n-2}$ and $I_{n-3}$ and 6 values of $\theta_{n}$, the resulting trellis has 384 states, which leads to a complex receiver. We use trellis state reduction techniques to reduce this complexity. In addition, we incorporate PSP into the MLSE receiver in order to include the channel estimation process. Finally, we add diversity combining to the receiver allowing for multiple receive antennas.

\section{A. Trellis State Reduction}

Reduced-state schemes are used to simplify the implemented trellis structure by exploiting in part the redundancy in the trellis. The frequency pulse truncation technique of [13] is used to truncate the frequency pulse of (5) used in defining the phase response $q(t)$. As the amplitudes of the tails of $g(t)$ are small, the resulting phase response can be truncated, thereby simultaneously reducing both the number of trellis states and the required number of matched filters, with little performance degradation. As this truncation actually reduces the correlativestate vector, it is a form of correlative state reduction [8]. In addition, we use the tilted phase approach of [1], [8] to achieve further complexity reduction. It is based on the decomposition model of [11]. This transforms the periodically time-varying trellis usually encountered in CPM into a time-invariant form that typically has only half as many phase states as the original trellis. It affects only the number of phase states and not the number of correlative states. This combination of techniques allows reduction of the numbers of both phase states and correlative states.

State reduction is achieved via a three step approach. The tilted phase approach is first used to reduce the number of states to 192 . This is followed by frequency pulse truncation which further reduces the number of states to 48 by reducing the number of correlative states. Finally, a further four-fold reduction is achieved by applying a selective frequency pulse excision process to obtain the final 12 state receiver.

1) Tilted Phase: For all CPM schemes, at any given symbol time, the value of $\theta_{n}$ can take only $p$ possible values, even though the total number of possible $\theta_{n}$ values may be either $p$ if $m$ is even, or $2 p$ if $m$ is odd [14]. When $m$ is odd (as considered here), the set of $2 p$ possible $\theta_{n}$ values is split into two subsets, with one subset used at even symbol times and the other at odd symbol times [14]. In particular, at odd symbol times, the phase state takes the values

$$
\theta_{n} \in\left\{0, \frac{2 \pi m}{p}, \frac{4 \pi m}{p}, \cdots, \frac{(p-1) 2 \pi m}{p}\right\}
$$

while at even symbol times, it takes the values

$$
\theta_{n} \in\left\{\frac{\pi m}{p}, \frac{3 \pi m}{p}, \frac{5 \pi m}{p}, \cdots, \frac{(2 p-1) \pi m}{p}\right\} .
$$

A proof of this is found in [14]. This results in a cyclically time variant trellis structure with a period of 2 symbol periods [14]. This time variant trellis can be transformed to an equivalent time invariant trellis called the tilted trellis [11]. The tilted trellis is arrived at by applying the phase offset

$$
v_{n}=v_{n-1}-\pi h(M-1)
$$

to "correct" the phase state values, where $v_{n-1}=0$ at $t=0$. Here we consider $m=1, p=3$ and $M=4$, resulting in $v_{n}=-\pi$ and $v_{n}=0$ for even and odd symbol periods, respectively. With this approach, the effective number of states required to decode PR-CPM is halved in each symbol interval without performance loss.

2) Frequency Pulse Truncation: Fig. 1 shows how frequency pulse truncation is applied to the PR-CPM signal. Fig. 1a, shows the effect of the phase response $q(t)$ on the transmitted signal for the PR-CPM $(L=4)$ encoding, starting from $t=0$ (current transmission) and tracing back through the previous symbols. From $t=0$ to $t=-4 T$, the value of $q(t)$ increases from 0 to 0.5 . This time span corresponds to the correlative state of the transmitted signal, as described by (3), which is defined by the three symbols $I_{n-1}, I_{n-2}$ and $I_{n-3}$ immediately preceding the current symbol, $I_{n}$. This is represented in Fig. 1a as the white region in the graph. For $t \leq-4 T$, the value of $q(t)$ remains constant at 0.5 , and the sum of all the symbols transmitted at and before this time frame defines the phase state $\theta_{n}$ of the transmitted signal. This is shown in Fig. 1a as the shaded region.

The precise implementation of frequency pulse truncation relies on the particular shape of $q(t)$. In particular, it can be seen from Fig. 1a that at $t=-3 T, q(t) \approx 0.5$. Therefore, the effect of the last symbol $I_{n-3}$ can be merged into $\theta_{n}$. This reduces the pulse time varying response length $L$ by 1 , which reduces the number of trellis states from 192 to 48 . Note that this makes no difference to the allowable values of the phase state $\theta_{n}$. 

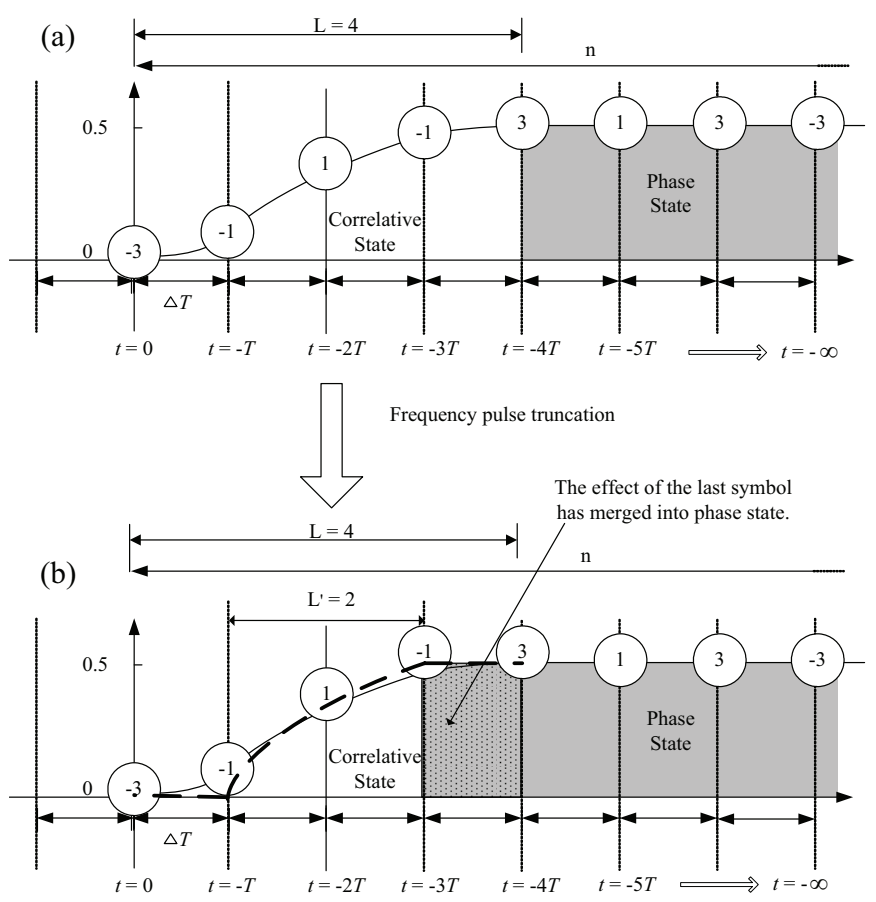

Fig. 1. The change in the correlative state and phase state upon application of frequency pulse truncation. Note that this is equivalent to performing a phase truncation as the phase pulse is simply the integral of the frequency pulse.

It can also be seen that at $t=-T, q(t)$ is small. Therefore, we excise the effect of the symbol at $t=-T$ on the correlative state. The resulting phase response length or correlative state definition is further reduced by 1 , giving $L^{\prime}=2$, which means that the number of trellis states at each symbol time is reduced to 12 . The new states corresponding to the reduced trellis are represented by $S=\left(\theta_{n}, I_{n-2}\right)$, based on which the VA then decodes the signal. Fig. 2 shows the reduced 12 state PR-CPM trellis. It also demonstrates the PR-CPM alternating phase states feature discussed earlier. This approach is called correlative state reduction, as the last element of the correlative state vector has been merged into the phase state vector [7] and the first element of the correlative state vector has been ignored. The final appearance of the phase response and the corresponding correlative and phase state are shown in Fig. 1b as the dashed trajectory.

\section{B. Channel Estimation using Per-Survivor Processing}

We now consider the Rayleigh fading channel and use PSP [10] to estimate the channel in the MLSE receiver. In a PSP receiver different channel responses are simultaneously estimated along the surviving paths associated with each state in the trellis of the VA. Each path maintains and updates its own channel estimate based on the corresponding hypothesized transmitted data sequence [10], [6], and that estimate is used to calculate branch metrics only for that path. The existence of individual gains for surviving paths means that each gain estimate is confined to the particular survivor path, along with

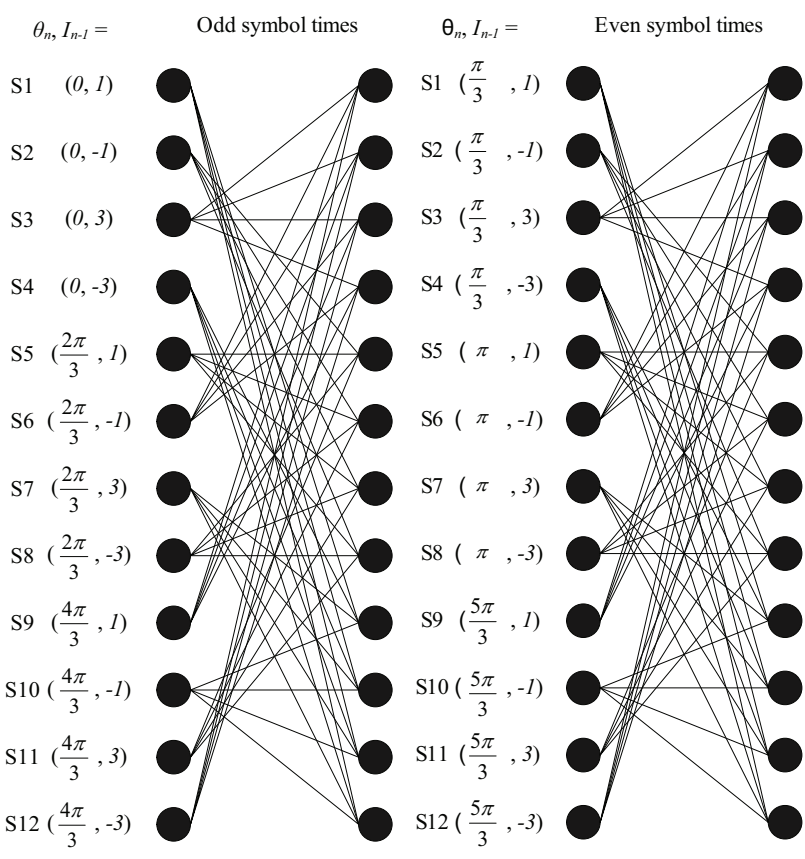

Fig. 2. Trellis example of the reduced state PR-CPM receiver.

its error. Unlike conventional MLSE, if the gain for a particular surviving path is corrupted with noise or distortion, then the rest of the surviving paths may not be affected. As decision making is based on the best surviving path, this error tends not to propagate through the decoding sequence. Further, as the gain is estimated based on the previous surviving paths, in general, the more reliable the surviving path is, the more reliable is the associated channel estimation. Hence PSP is suitable for time-varying channels and achieves significant reduction in the effects of error propagation. The drawback of such PSP-based MLSE receivers is their computational complexity as channel estimation is required for each survivor path rather than requiring only one global channel estimate.

It can be shown that the correlation function between the received signal $r(t)$ and the tentative decoded signal $s(t)$, $\operatorname{corr}(r, s)$, is a noisy estimate of the channel gain. Hence we can obtain a more accurate estimate of the channel gain by reducing the noise component of the correlation function. This can be done by averaging, possibly in a weighted manner, $N$ successive estimates to obtain a better gain estimate, where the choice of $N$ is based on the Doppler spread.

In this paper, a least mean squared error estimate of the channel gain is used. We assume a Jakes' Doppler spectrum and estimate the channel gain as the weighted sum

$$
\begin{aligned}
h(n T)= & w_{0} \operatorname{corr}(r, s)+w_{1} h((n-1) T)+w_{2} h((n-2) T) \\
& +\cdots+w_{N} h((n-N+1) T),
\end{aligned}
$$

where $\boldsymbol{w}^{T}=\left[w_{0}, w_{1}, w_{2}, \cdots, w_{N}\right]$ is defined by the YuleWalker equation [9]

$$
\boldsymbol{w}=\boldsymbol{R}_{y}^{-1} \boldsymbol{p} .
$$


The $(i, j)^{t h}$ component of the covariance matrix, $\boldsymbol{R}_{y}$, is given by

$$
\boldsymbol{R}_{y_{i, j}}=2 \gamma_{s} J_{0}\left(2 \pi f_{d(\max )} T(i-j)\right)+\delta((i-j), 0),
$$

where $f_{d(\max )}$ is the maximum Doppler frequency, $\gamma_{s}$ is the signal to noise ratio $(S N R)$ of the channel and $J_{0}$ is the zero order Bessel function. The cross correlation vector $\boldsymbol{p}$ (of length $L)$ has $i^{\text {th }}$ component given by

$$
\boldsymbol{p}_{i}=\sqrt{2 \gamma_{s} J_{0}\left(2 \pi f_{d(\max )} T(i+1)\right)}, \quad i=1, \cdots, L .
$$

This takes into account the speed of fading, and weights the successive estimates accordingly. The weighted sums provide channel gain estimates for each surviving path, which are incorporated into the branch metrics given by

$$
B(n T)=\operatorname{Re}\left\{r(n T) h^{*}(n T) s^{*}(n T)\right\},
$$

where ** denotes complex conjugate and $R e\{\}$ denotes the real part. By setting the weights $\boldsymbol{w}=1$, we obtain a simple average, which can be used, but leads to inferior performance.

\section{Diversity Combining}

We now look at the impact of receiver diversity through the use of multiple, $N_{r}$, receive antennas. Three standard approaches to diversity combining are considered, namely selection combining (SC), equal gain combining (EGC) and maximum ratio combining (MRC). For selection combining, the strongest received signal from one of the antennas is chosen as the received signal. PSP is performed using the selected received signal.

EGC and MRC perform PSP on each received signal stream prior to actual diversity combining. EGC uses a phase coherent unweighted linear sum of the received signals. The required phase estimates are directly provided by the PSP process. MRC uses a phase coherent weighted linear sum of the received signals [2]. The weights are the ratio of the channel gain magnitude to the noise energy [2]. Hence, MRC requires both the estimated channel phase and magnitude from PSP. As both EGC and MRC perform PSP on each received signal, they have higher complexity than SC. PSP of the received signal streams can be done in parallel to avoid increased latency. Due to PSP providing full channel state estimates, EGC and MRC have similar complexity.

\section{Simulation Results}

We use simulation to evaluate performance. $N_{\text {win }}=10$ was chosen as the decision depth in the VA as it resulted in very similar performance to large depths such as $N_{\text {win }}=30$. SNR is measured as bit energy, $E_{b}$, divided by noise power spectral density, $N_{0}$.

Fig. 3 shows the bit error rate (BER) performance of the reduced state MLSE receiver in an AWGN channel. It can be seen that there is a slight degradation in error performance as the number of trellis states is reduced from 192 to 48 . This is expected as an approximation is made in the frequency pulse truncation process, and thus, a small extra error is introduced

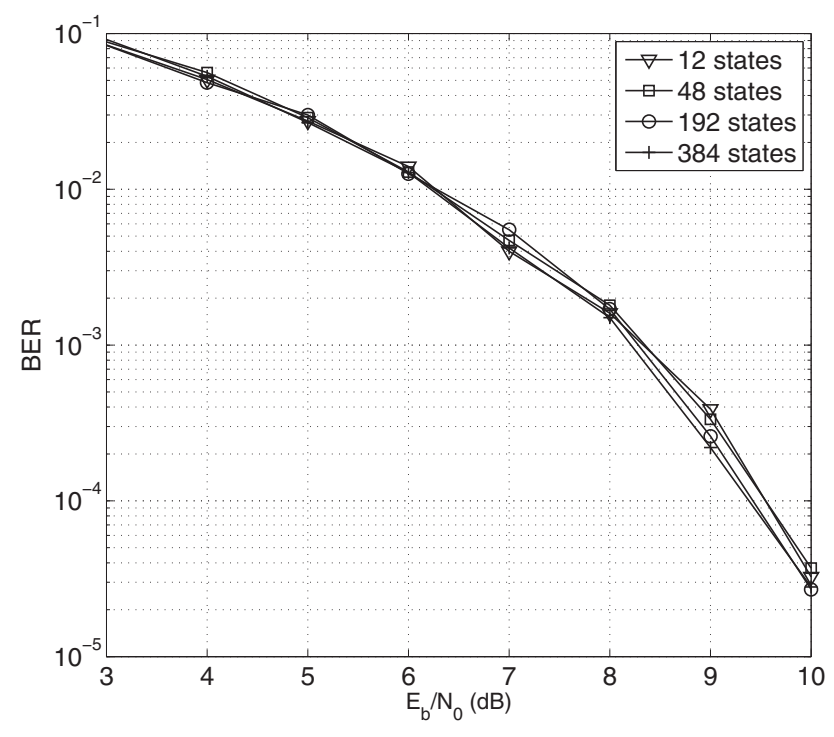

Fig. 3. MLSE receiver performance using 12, 48, 192 and 384 states on an AWGN channel.

into the decoding process. Similarly, the state reduction from 48 to 12 states also leads to a further small error performance degradation. As there is only a very small degradation in performance it is clear that the significant effects of the correlative state are retained.

The BER curve for the PSP-based MLSE receiver in a fading environment is presented in Fig. 4 for averaging length $N=5$ in (11) and Doppler frequencies of $5 \mathrm{~Hz}, 40 \mathrm{~Hz}$ and $80 \mathrm{~Hz}$. Note that a longer integration length would result in better channel estimation and slightly improved BER. As expected, PSP using a weighted sum significantly outperforms PSP with standard averaging and no PSP at Doppler frequencies above $5 \mathrm{~Hz}$. Performance could be further improved by adaptively changing the weight coefficients based on minimizing the error in a mean square sense. This is the approach used in [16] for linear modulations.

The BER performance with diversity combining is shown in Fig. 5 for a Doppler frequency of $80 \mathrm{~Hz}$. As expected, MRC provides the best performance followed by EGC and SC. The largest gains are obtained by increasing from 1 to 2 receive antennas.

\section{Conclusions}

The paper has described a practical design for a PSPbased MLSE receiver for PR-CPM which is applicable to public safety narrow band deployment around the world. The modulation described here is characterized by a long impulse response. Nevertheless, state reduction has been successfully applied which minimizes the resources required for DSP or FPGA implementation whilst maintaining excellent receiver performance in the mobile environment.

Diversity has also been examined and the results show the deployment of diversity can substantially improve the faded 
performance assuming the diversity channels are sufficiently de-correlated. The integration of PSP with MRC is the key practical advance in this work. As PSP provides channel estimates and hence the diversity coefficients at each state for each received signal component, diversity combining using EGC and MRC have similar complexity, allowing the superior performance of MRC for negligible additional complexity. Moreover, PSP resolves any phase ambiguities [16]. Spatial diversity represents the obvious method of acquiring these gains and modern public safety base stations are becoming available with this capacity.

\section{ACKNOWLEDGMENT}

We acknowledge the assistance of Prof. J. K. Cavers who developed the weighted sum approach in (11) and shared it with us in a personal communication. Unfortunately, it is not available publicly.

\section{REFERENCES}

[1] J. B. Anderson, T. Aulin, and C.-E. Sundberg, "Digital Phase Modulation". New York: Plenum Press, 1986.

[2] D. G. Brennan,"Linear diversity combining techniques," Proc. IEEE, vol. 91, pp.331-356, 2003.

[3] M. Kalkan, "CPM performance with diversity in mobile radio," in Proc. 7th Mediterranean Electrotechnical Conference, vol. 1, pp. 3234, 1994.

[4] M. J. Miller, "Detection of CPFSK signals using per survivor processing," in Proc. MILCOM, 1998.

[5] F. A. Monteiro and A. J. Rodrigues, "Phase Error Resilience to I/Q Mismatch of a Simplified CPM Receiver,' IEEE Microwave and wireless components letters, vol. 15, Sep. 2005.

[6] M. J. Omidi, S. Pasupathy and P. G. Gulak, "Joint data and Kalman estimation for Rayleigh fading channels," Wireless Pers. Commun., vol. 10, pp. 319-339, 1999.

[7] E. S. Perrins, "Reduced complexity detection methods for continuous phase modulation,'Ph.D. Thesis, Brigham Young University, Dec. 2005.

[8] E. Perrins and M. Rice, "Reduced-complexity detectors for Multi-h CPM in aeronautical telemetry," IEEE Trans. Aerospace and Electronic Systems, vol. 43, pp. 286-300, 2007.

[9] J. G. Proakis, "Digital Communications", 4th Edition, New York: McGraw-Hill, 2001.

[10] R. Raheli, A. Polydoros and T. Ching-Kae, "Per-Survivor Processing: a general approach to MLSE in uncertain environments," IEEE. Trans. Commun., vol. 43, no. 234, pp. 354-364, Feb.-Mar.-Apr. 1995.

[11] B. E. Rimoldi,"A decomposition approach to CPM, "IEEE Trans. Inform. Theory, Vol. 34, No. 2, pp. 260-270, Mar. 1988.

[12] C. E. Sundberg, "Continuous phase modulation," IEEE. Commun. Mag. vol. 24 , pp. $25-38,1986$.

[13] A. Svensson, C.-E. Sundberg and T. Aulin, "A Class of ReducedComplexity Viterbi Detectors for Partial Response Continuous Phase Modulation," IEEE. Trans. Commun., vol. 32, No. 10, pp. 1079-1087, Oct. 1984.

[14] W. Tang, "A receiver for continuous phase modulation in Walsh signal space, Ph.D. Thesis, University of Winnipeg, Manitoba, Canada, Sept. 1998.

[15] TIA, "APCO project 25 system and standards definition," TIA/EIA Telecommunications Systems Bulletin, TSB102-A 1995.

[16] G. M. Vitetta and D. P. Taylor, "Maximum likelihood decoding of uncoded and coded PSK signal sequences transmitted over Rayleigh flat-fading channels", "IEEE Trans. Commun., Vol. 43, pp. 2750-2758, Nov. 1995.

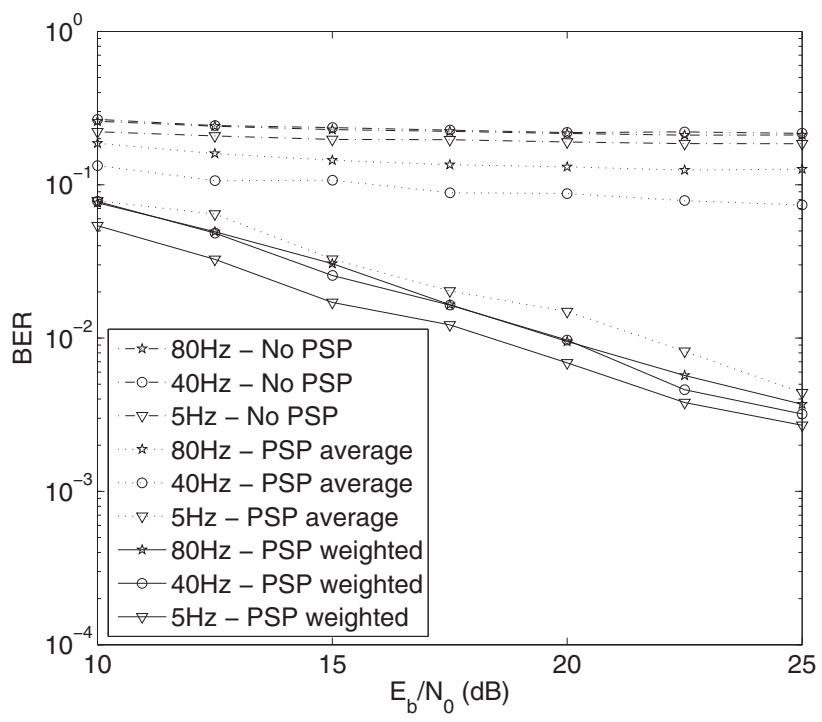

Fig. 4. BER performance of 12-state PSP-MLSE receiver using standard averaging and weighted sum compared to that of the MLSE receiver without PSP in Rayleigh fading channel. Symbol time $T=1 / 6000$. Doppler frequencies $5 \mathrm{~Hz}, 40 \mathrm{~Hz}$ and $80 \mathrm{~Hz}$.

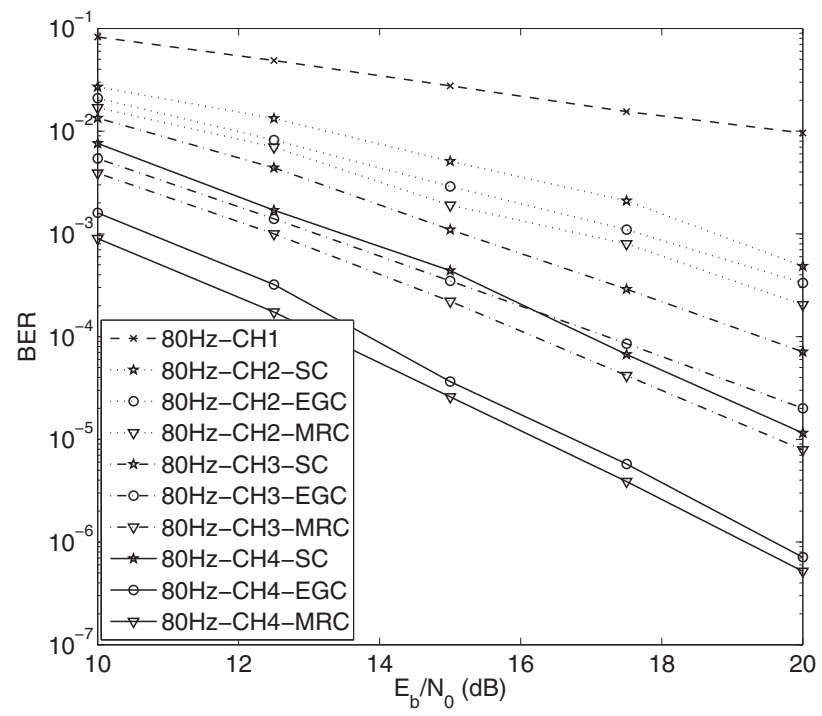

Fig. 5. BER performance comparison for 12-state PSP-MLSE receiver using no diversity $(\mathrm{CH} 1)$, dual $(\mathrm{CH} 2)$, triple $(\mathrm{CH} 3)$ and quadruple $(\mathrm{CH} 4)$ diversity systems in Rayleigh fading channel at Doppler frequency of $80 \mathrm{~Hz}$. Symbol time $T=1 / 6000$ 